\title{
Respiratory Frequency Estimation from Accelerometric Signals Acquired by Mobile Phone in a Controlled Breathing Protocol
}

\author{
F. Landreani ${ }^{1}$, A. Martin-Yebra ${ }^{1,2}$, C. Casellato ${ }^{1}$, E. Pavan ${ }^{1}$, C. Frigo ${ }^{1}$, P-F. Migeotte ${ }^{3}$, A. Faini ${ }^{4}$, \\ G.Parati ${ }^{4,5}$, E.G. Caiani ${ }^{1}$ \\ ${ }^{1}$ Dipartimento di Elettronica, Informazione e Bioingegneria, Politecnico di Milano, Milan, Italy \\ ${ }^{2}$ Aragón Institute of Engineering Research, IIS Aragón, Universidad de Zaragoza, Zaragoza, Spain \\ ${ }^{3}$ Université Libre de Bruxelles, Brussels, Belgium \\ ${ }^{4}$ Istituto Auxologico Italiano Ospedale San Luca, Milan, Italy \\ ${ }^{5}$ Department of Medicine and Surgery, Università di Milano-Bicocca, Milan, Italy
}

\begin{abstract}
The aim of this work was to test if the smartphone's embedded triaxial accelerometer can be used to extract respiratory frequency information from the chest movements during a controlled breathing protocol. Respiratory signals from 10 young volunteers were recorded simultaneously, by two smartphones (iPhone $4 \mathrm{~s}$ and 6 ; sampling frequency $\sim 100 \mathrm{~Hz}$ ), positioned one on the sternum and one on the belly, while in supine posture. At the same time, a belt transducer was used to acquire the reference respiratory signal. A controlled breathing protocol, consisting of four consecutive phases of 12 respiratory cycles each (respiratory frequencies at 0.25, 0.17, 0.125 and $0.1 \mathrm{~Hz}$ ), was imposed through the visualization of a moving bar on a display. After low-pass filtering $(f c=0.5 \mathrm{~Hz})$, the respiratory signal was obtained from both smartphones, and respiratory frequency derived for each phase. Compared to the belt transducer, the resulting error was lower than $2 \%$ for each imposed respiratory frequency, for both smartphones' positions, with better results obtained for the smartphone positioned above the belly.
\end{abstract}

\section{Introduction}

The use of mobile phones is increasing ubiquitously, and individuals are assuming a more active role in monitoring and managing their health and wellness. The introduction of this device for clinical applications and self-tracking has the potential to change the way healthcare is delivered [1].

In healthcare applications, the feasibility to use smartphone's accelerometers have been already tested to monitor subject's activity [2], [3]. Moreover, its potential use for heartbeat detection it has been already demonstrated: when properly positioning the smartphone on the chest, close to the heart apex, the vibrations due to the heart contraction are recorded (i.e., seismocardiography), from which to derive the beat-tobeat duration measurements [4], [5].

The use of the accelerometers to record the respiratory activity has been already proposed [6], [7]. The accelerometers are embedded into a flexible belt, which can be preferable placed around the thorax at the level of the heart apex, capturing the movements of the chest derived from the subject respiration. As respiration is actually one of the most monitored vital signs, accelerometers represent an easy and simple alternative method to measure the respiratory movements in an unobtrusive way, in particular in an outpatient environment.

In this context, the smartphone's embedded accelerometers could represent an alternative tool to record the respiratory signal, offering the possibility of recording respiratory activity in a non-invasive way using a hand-held device. This work aimed at exploring the feasibility of the smartphone's triaxial accelerometer to monitor the respiratory activity from two possible locations on the subject's torso, following a controlled breathing protocol, and to test the accuracy of the estimated respiratory frequencies compared to a belt reference.

\section{Experimental set-up and study population}

Two mobile phones (iPhone4s and 6s, sampling frequency $\sim 100 \mathrm{~Hz}$ ) were used to acquire the accelerometric signal to derive the respiratory waveform 
(RW). One device was positioned approximately at the heart apex, above the sternum (POS1), while the other was placed above the belly (POS2). The reference respiratory waveform was simultaneously acquired by a belt $\left(\mathrm{RW}_{\mathrm{b}}\right)$ transducer (Pneumotrace II; AD Instruments), that measured the changes in the abdomen and thoracic circumference, thus following the inhalation and expiration, and then used to derive the breathing rate.

In addition, a 6-lead electrocardiogram (ECG) (Nexfin HD monitor, BMEYE, Amsterdam) sampled at $1000 \mathrm{~Hz}$ was simultaneously acquired. The two smartphones were synchronized at the beginning and at the end of the protocol, by tapping on the subject shoulder. In this way, a motion artefact was sensed concurrently by both devices and by the ECG, thus allowing temporal alignment with the belt transducer signal. Ten healthy volunteers took part in this pilot study (Age: $22.3 \pm 1.4$ years; Body Mass Index: $\left.21.9 \pm 1.2 \mathrm{Kg} / \mathrm{m}^{2}\right)$. The experimental procedures described in this paper were in agreement with the ethics principles defined in the Helsinki Declaration of 1975, as revised in 2013. Ethical committee approval of the Université Libre de Bruxelles, Hopital Erasme, was obtained prior to the study. The subjects laid on a bed in supine position and performed a controlled breathing protocol (CBP), by following a moving bar on a display. This bar oscillates at four different frequencies fixed at $0.25,0.17,0.125$ and 0.1 $\mathrm{Hz}$, respectively, for 12 cycles each. The CBP was set so that inspiration and expiration periods were of the same duration, and the entire recording lasted approximately 6 minutes.

\section{Methods}

\subsection{Pre-processing step}

As can be observed in Fig. 1, the signal acquired from the triaxial accelerometer using the smartphone contains information from both respiratory and cardiac activities, mainly visible along the longitudinal ( $\mathrm{Y}$-axis) and the antero-posterior (Z-axis) components. Periodic deflections due to respiration were clearly visible along the longitudinal axis, especially for the mobile phone at POS2. Accordingly, only this longitudinal component of the accelerometric signal from both smartphones (named as $\mathrm{RW}_{\mathrm{POS} 1}, \mathrm{RW}_{\mathrm{POS} 2}$ ) was taken into consideration for further analysis. The baseline wandering of the accelerometric signal due to the respiratory activity was preserved with a low-pass filter with a cut-off frequency fixed at $0.5 \mathrm{~Hz}$, using a $4^{\text {th }}$ Butterworth filter, to remove cardiac activity. In addition, each respiratory phase of the CBP was visually identified, to separately proceed in deriving respiratory frequency for each phase, both in time and frequency domain.
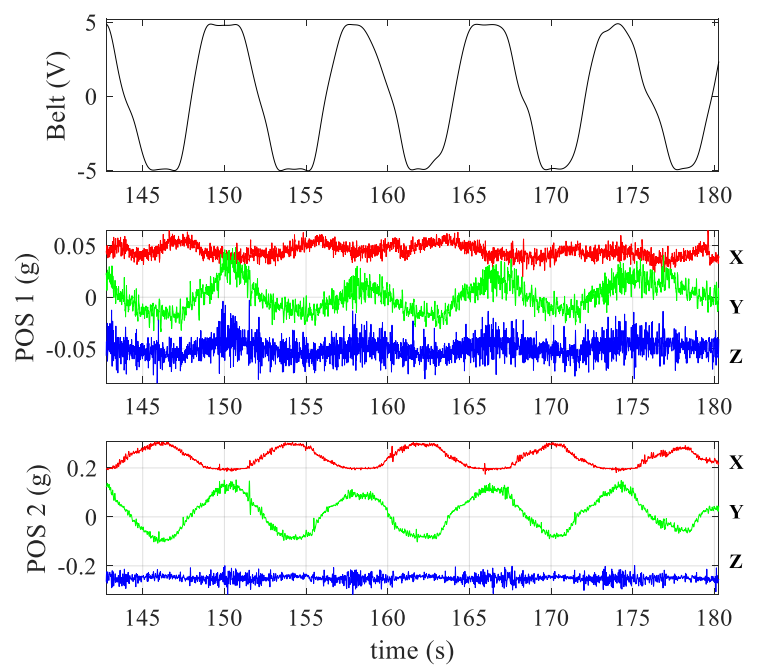

Figure 1. Example of the three accelerometric orthogonal components $(\mathrm{X}, \mathrm{Y}, \mathrm{Z})$ recorded by the smartphone in POS1 and POS2. The chest wall movements for respiratory activity were mainly visible along the longitudinal (Y-axis) component with respect to the other two directions.
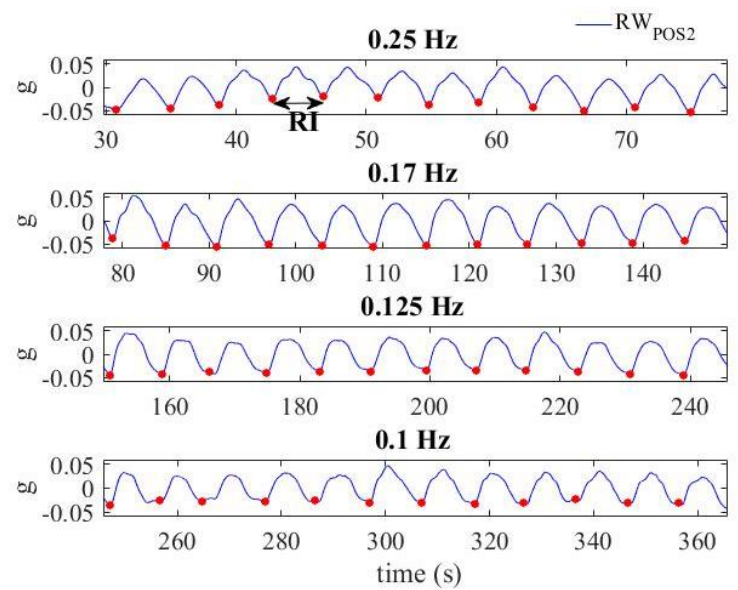

Figure 2. Example of low-pass filtered longitudinal component of the accelerometric signal, representing the respiratory activity, recorded at POS2 during controlled breathing at different frequencies $(0.25$, $0.167,0.125$ and $0.1 \mathrm{~Hz}$ ). The detected valleys (red dots) defined the respiratory intervals (RI).

\subsection{Time domain analysis}

Respiratory intervals (RI), each defined as the distance between two consecutive minima, were automatically identified by detecting the minimum of the RW signals (Figure 2) and of the belt signal, using an algorithm implemented in Matlab R2016b (The Mathworks). The detection procedure was achieved by searching the valley using a time-moving window spanning the $70 \%$ of the 
duration of the preceding cycle. Respiratory intervals from the belt transducer ( $\left.\mathrm{RI}_{\text {Belt }}\right)$ and the smartphones $\left(\mathrm{RI}_{\text {smart }}\right)$ were used to calculate the respiratory frequency (RF) as the inverse of the average of all the RI measured in each protocol phase. Results obtained from the smartphones and the belt reference in each phase were compared and the relative error $\left(\varepsilon_{\mathrm{T}} \%\right)$ was evaluated as:

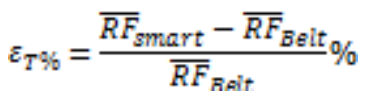

\subsection{Frequency domain analysis}

In order to proceed with spectral analysis, due to the unequally sampled data the Lomb-Scargle periodogram was utilized: it is suited for weak periodic signals in unevenly sampled data. The Lomb-Scargle power spectral density (PSD LS) estimate was computed on each previously selected respiratory phase, from which the respiratory frequency was derived as the frequency

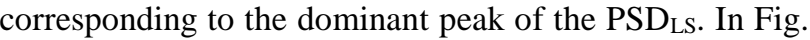
3 , the periodograms obtained from the respiratory signals corresponding to the protocol phase with dominant frequency imposed at $0.17 \mathrm{~Hz}$, acquired by the belt transducer $\left(\mathrm{LS}_{\mathrm{B}}\right)$ and the mobile phones $\left(\mathrm{LS}_{\mathrm{POS} 1}, \mathrm{LS}_{\mathrm{POS} 2}\right)$ are shown over imposed. It is possible to observe their very good correspondence around the dominant frequency. Also in this case, for each CBP phase, the relative error $\left(\varepsilon_{\mathrm{F}} \%\right)$ between the respiratory frequencies extracted by the $\mathrm{PSD}_{\mathrm{LS}}$ periodograms, from the smartphones ( $\left.\overline{(R F}_{L S \text { amart }}\right)$ and from the belt transducer $\left(\overline{R F}_{\text {LS Eelt }}\right)$, was calculated as:

$$
\varepsilon_{F \% 6}=\frac{\overline{R F}_{L S \text { smart }}-\overline{R F}_{L S \text { Beit }}}{\overline{R F}_{L S \text { Beit }}} \%
$$

\subsection{Statistical analysis}

Bland-Altman analysis was applied to compare the frequency extracted by the belt signal and those derived from the smartphones, separately for POS1 and POS2, for both the results obtained in the time and frequency domains.

\section{Results}

Of the 10 acquired subjects, one needed to be excluded due to problems in the signal acquired by the belt reference. In the time domain analysis, out of the remaining 9 subjects, feasibility of the automated analysis was $7 / 9(78 \%)$ for POS2 and 5/9 (55\%) for POS1, where discarded signals were related to low signal-to-noise ratio preventing the automated detection of valleys in the signal. The relative error compared to the belt-derived

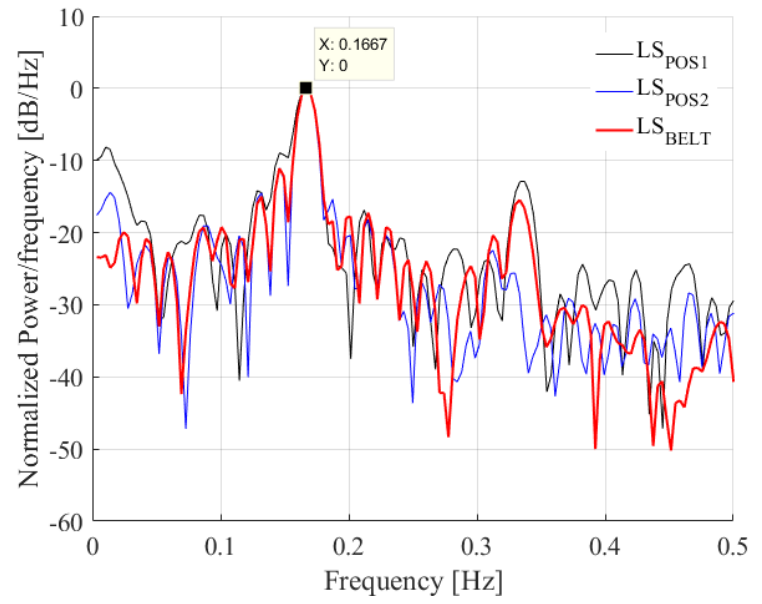

Figure 3. Example of Lomb-Scargle periodograms obtained from the respiratory signals, relevant to the CBP phase with the dominant frequency set at 0.17 $\mathrm{Hz}$, by the belt transducer (red), compared to that derived from the mobile phones located at POS1 (black) and POS2 (blue).

results was lower than $1.5 \%$ for each phase of the $\mathrm{CBP}$, for both smartphone positions, with better results obtained in POS2 (see Table 1). For the frequency domain analysis, the feasibility reached $100 \%$ for POS1 and POS2. The relative error compared to the belt-derived results was lower that $1.5 \%$ (see Table 2).

In all cases, Bland-Altman analysis showed no significant biases and narrow limits of agreement, with the best results obtained for the respiratory frequencies extracted in POS2 for all four CBP phases (see Table 3).

\section{Discussion and conclusions}

In this pilot study, the feasibility of using the triaxial accelerometer embedded in the smartphone to derive the respiratory activity was tested, together with its accuracy in respiratory frequency estimation compared to a thoracic belt reference.

Two positions of the smartphone during acquisition were simultaneously tested to simulate potential easy to access points by the user. A respiratory breathing protocol, including 12 cycles for each of the four frequencies imposed, was utilized to derive results in a wide range of possible respiratory frequencies (from 0.1 $\mathrm{Hz}$ to $0.25 \mathrm{~Hz}$ ). The respiratory frequencies were computed in both time and frequency domains from the low-pass longitudinal component of the accelerometric signal, in order to test two possible computational methodologies.

The time domain approach required the detection of valleys characterizing the different breathing cycles. Due to noisy signals, best feasibility of this approach was 
Table 1. Average respiratory frequencies (mean $\pm \mathrm{SD}$ ) and relative errors obtained by belt reference $(\mathrm{RWb})$ and the smartphones in POS1 and POS2 (RW POS1, $\mathrm{RW}_{\mathrm{POS} 2}$, at each respiratory frequency (RF) imposed by the CBP, obtained by time domain analysis

\begin{tabular}{|c|c|c|c|}
\hline $\begin{array}{c}\mathrm{RF} \\
{[\mathrm{Hz}]}\end{array}$ & $\mathrm{RWb}$ & $\begin{array}{c}\text { RWPOS1 } \\
\varepsilon_{\mathrm{T} \%}\end{array}$ & $\begin{array}{c}\text { RWPOS2 } \\
\varepsilon_{\mathrm{T} \%}\end{array}$ \\
\hline 0.25 & $0.251 \pm 0.003$ & $\begin{array}{c}0.249 \pm 0.002 \\
0.25\end{array}$ & $\begin{array}{c}0.252 \pm 0.002 \\
0.33\end{array}$ \\
\hline 0.17 & $0.168 \pm 0.003$ & $\begin{array}{c}0.168 \pm 0.002 \\
1.12\end{array}$ & $\begin{array}{c}0.167 \pm 0.002 \\
1.08\end{array}$ \\
\hline 0.125 & $0.126 \pm 0.001$ & $\begin{array}{c}0.125 \pm 0.001 \\
0.99\end{array}$ & $\begin{array}{c}0.126 \pm 0.001 \\
0.59\end{array}$ \\
\hline 0.1 & $0.101 \pm 0.001$ & $\begin{array}{c}0.101 \pm 0.002 \\
0.63\end{array}$ & $\begin{array}{c}0.101 \pm 0.001 \\
0.42\end{array}$ \\
\hline
\end{tabular}

Table 2. Average respiratory frequencies (mean $\pm \mathrm{SD}$ ) and relative errors obtained by belt reference (RWb) and the smartphones in POS1 and POS2 (RWPOS1, RWPOS2, at each respiratory frequency (RF) imposed by the CBP, obtained by frequency domain analysis.

\begin{tabular}{|c|c|c|c|}
\hline $\begin{array}{c}\text { RF } \\
{[\mathrm{Hz}]}\end{array}$ & RWb & $\begin{array}{c}\mathrm{RW}_{\mathrm{POS} 1} \\
\varepsilon_{\mathrm{F} \%} \%\end{array}$ & $\begin{array}{c}\mathrm{RW}_{\mathrm{POS} 2} \\
\varepsilon_{\mathrm{F} \%}\end{array}$ \\
\hline 0.25 & $0.251 \pm 0.003$ & $\begin{array}{c}0.251 \pm 0.002 \\
0.51\end{array}$ & $\begin{array}{c}0.249 \pm 0.002 \\
0.85\end{array}$ \\
\hline 0.17 & $0.167 \pm 0.001$ & $\begin{array}{c}0.168 \pm 0.002 \\
0.66\end{array}$ & $\begin{array}{c}0.166 \pm 0.002 \\
1.25\end{array}$ \\
\hline 0.125 & $0.125 \pm 0.001$ & $\begin{array}{c}0.124 \pm 0.001 \\
0.74\end{array}$ & $\begin{array}{c}0.128 \pm 0.001 \\
0.26\end{array}$ \\
\hline 0.1 & $0.101 \pm 0.001$ & $\begin{array}{c}0.097 \pm 0.008 \\
0.49\end{array}$ & $\begin{array}{c}0.101 \pm 0.001 \\
0.43\end{array}$ \\
\hline
\end{tabular}

Table 3. Results of Bland-Altman analysis (bias \pm 2SD) obtained by the comparison of the frequency extracted by the LS periodograms of the belt signal and those derived from the smartphones.

\begin{tabular}{|c|c|c|c|c|}
\hline $\begin{array}{c}\mathrm{RF} \\
{[\mathrm{Hz}]}\end{array}$ & POS1 & $\varepsilon \%$ & POS2 & $\varepsilon \%$ \\
\hline 0.25 & $-0.001 \pm 0.0050$ & 0.98 & $-0.001 \pm 0.006$ & 0.8 \\
\hline 0.17 & $0.002 \pm 0.004$ & 1.6 & $0.002 \pm 0.003$ & 1.5 \\
\hline 0.125 & $0.001 \pm 0.002$ & 0.99 & $0.001 \pm 0.001$ & 0.97 \\
\hline 0.1 & $0.001 \pm 0.002 \quad 0$ & 0.96 & $0.0004 \pm 0.002$ & 0.7 \\
\hline
\end{tabular}

limited to $78 \%$ at POS2. The frequency domain approach resulted in $100 \%$ feasibility and faster computation. Both in time and frequency domains, the average respiratory frequency from both locations, belly and sternum, was estimated with an error lower than $1.5 \%$ with respect to the ones measured by the reference signal, which well corresponded to the imposed frequencies along the protocol. From the Bland-Altman analyses, it was possible to appreciate that the relative error remained low either with the mobile phone positioned at the sternum or above the belly $(<2 \%)$, with the position above the belly resulting in the lowest relative percentage error. The utilization of the smartphone's accelerometer to detect respiratory frequency appeared feasible, in particular if positioned above the belly, and accurate compared to a belt reference transducer. This methodology could represent the basis for apps aiming at tracking the respiratory frequency, with applications that could reveal useful for patients with chronic conditions, like heart failure.

\section{Acknowledgements}

This study was supported by the Italian Space Agency (contract "3D ballistocardiography in microgravity" 2013-064-R.0, Principal Investigator EG Caiani). P.F. Migeotte was supported by the Belgian Federal Science Policy Office (BELSPO) via the ESA PRODEX program.

\section{References}

[1] N. Bruining, E. Caiani, C. Chronaki, P. Guzik, and E. van der Velde, "Acquisition and analysis of cardiovascular signals on smartphones: potential, pitfalls and perspectives By the Task Force of the e-Cardiology Working Group of European Society of Cardiology," Eur. J. Prev. Cardiol., vol. 21, no. 2 suppl, pp. 4-13, Nov. 2014.

[2] L. Tong, Q. Song, Y. Ge, and M. Liu, "HMM-Based Human Fall Detection and Prediction Method Using TriAxial Accelerometer," IEEE Sens. J., vol. 13, no. 5, pp. 1849-1856, May 2013.

[3] A. Bayat, M. Pomplun, and D. A. Tran, "A Study on Human Activity Recognition Using Accelerometer Data from Smartphones," Procedia Comput. Sci., vol. 34, pp. 450-457, Jan. 2014.

[4] F. Landreani et al., "Ultra-short-term heart rate variability analysis on accelerometric signals from mobile phone," in 2017 E-Health and Bioengineering Conference (EHB), 2017, pp. 241-244.

[5] F. Landreani et al., "Beat-to-beat heart rate detection by smartphone's accelerometers: Validation with ECG," in 2016 38th Annual International Conference of the IEEE Engineering in Medicine and Biology Society (EMBC), 2016, pp. 525-528.

[6] P. D. Hung, S. Bonnet, R. Guillemaud, E. Castelli, and P. T. N. Yen, "Estimation of respiratory waveform using an accelerometer," in 2008 5th IEEE International Symposium on Biomedical Imaging: From Nano to Macro, 2008, pp. 1493-1496.

[7] M. Jafari Tadi, T. Koivisto, M. Pänkäälä, and A. Paasio, "Accelerometer-Based Method for Extracting Respiratory and Cardiac Gating Information for Dual Gating during Nuclear Medicine Imaging," Int. $J$. Biomed. Imaging, vol. 2014, 2014.

Address for correspondence.

Federica Landreani.

DEIB, Politecnico di Milano, Piazza Leonardo da Vinci, 32, 20133, Milano, Italy

federica.landreani@polimi.it 